\title{
Clinical-
}

Pathologic

Conference

\section{Clinical-pathologic conference in general thoracic surgery: Pulmonary artery fibrohistiocytic tumor in a child}

Thorsten Walles, MD

Paolo Macchiarini, MD, PhD

From Hannover Medical School, Hannover, Germany.

\section{Participants \\ From Hannover Medical School, Han- nover, Germany.}

Thoracic and Cardiovascular Surgery Dr Thorsten Walles

Dr Paolo Macchiarini

Dr Axel Haverich

Pathology

Dr Hans Kreipe

Diagnostic Radiology

Dr Michael Galanski

Pediatric Pneumology

Dr Joachim Freihorst

\section{Case Presentation}

Dr Walles. The patient was a 10-year-old boy weighing $29.1 \mathrm{~kg}$ (50th percentile) and was $160.5 \mathrm{~cm}$ (50-75th percentile) tall. He was complaining of night sweats, inspiratory pain located under the right costal arches and radiating to the right shoulder, and progressive exhaustion, exertional dyspnea, and weight loss for 1 month. His medical history was unremarkable, and there was no evidence of tuberculosis exposition or tumor disease in his family records. On an outpatient basis, an initial diagnosis of exertional bronchospastic obstruction was made, and he was given bronchodilator inhaled agents without success. Because of this, a plain chest radiograph was made showing a shadow in the right upper thoracic cavity, interpreted initially as pleuritis. To further clarify the diagnosis, a computed tomograph (CT) of the chest was made that showed a tumor mass located in the right hilus, with enlarged lymph nodes in the ipsilateral upper mediastinum. A nuclear magnetic resonance image ruled out additional abdominal masses or lymph nodes. Perhaps at this point we could have the pediatric pneumologist present the findings of the patient at hospital admittance.

Dr Freihorst. On admission the patient showed slight congestion of the superficial veins over the chest and abdomen. He was a little tachypneic, with mild intercostal retractions, but had no cyanosis, and breath sounds were vesicular, with minimal wheezing over the right lung. Physical examination was unremarkable otherwise. A sonogram of the neck showed enlarged lymph nodes at the right prescalenic area, and one of them was surgically resected for histologic diagnosis. Serum $\beta$-human chorionic gonadotropin hormone and $\alpha$-fetoprotein markers and urinary levels of catecholamine were normal. Fiberoptic bronchoscopy showed slight inflammation of the right upper lobe takeoff but no endoluminal tumor or other significant abnormalities, and a bronchoalveolar lavage and a transbronchial biopsy of the enlarged right paratracheal lymph nodes were performed.

Dr Kreipe. The pathologic-anatomic examination of the lymph node revealed no evidence of malignancy but showed a nonspecific inflammatory reaction. The same was found in the transbronchial biopsy samples.

Dr Freihorst. Transthoracic echocardiography found a distal obstruction of the right pulmonary artery, resulting in a moderate right heart strain. A subsequent right heart catheterization was attempted but was impossible to complete because of the total obstruction of the right pulmonary artery.

Dr Galanski. Lung scintigraphy showed normal ventilation patterns but a complete absence of perfusion of the right lung. A second thorax CT confirmed the endoluminal obstruction of the right pulmonary artery (Figure 1) and enlarged lymph nodes and showed normal ventilation patterns. As a new finding, a rich bronchial arterial collateral network provided peripheral lung perfusion (Figure 2).

Dr Walles. Thus, we had a 10-year-old boy with a 1-month history of exertional dyspnea and exhaustion due to a total tumoral obstruction of his pulmonary artery 


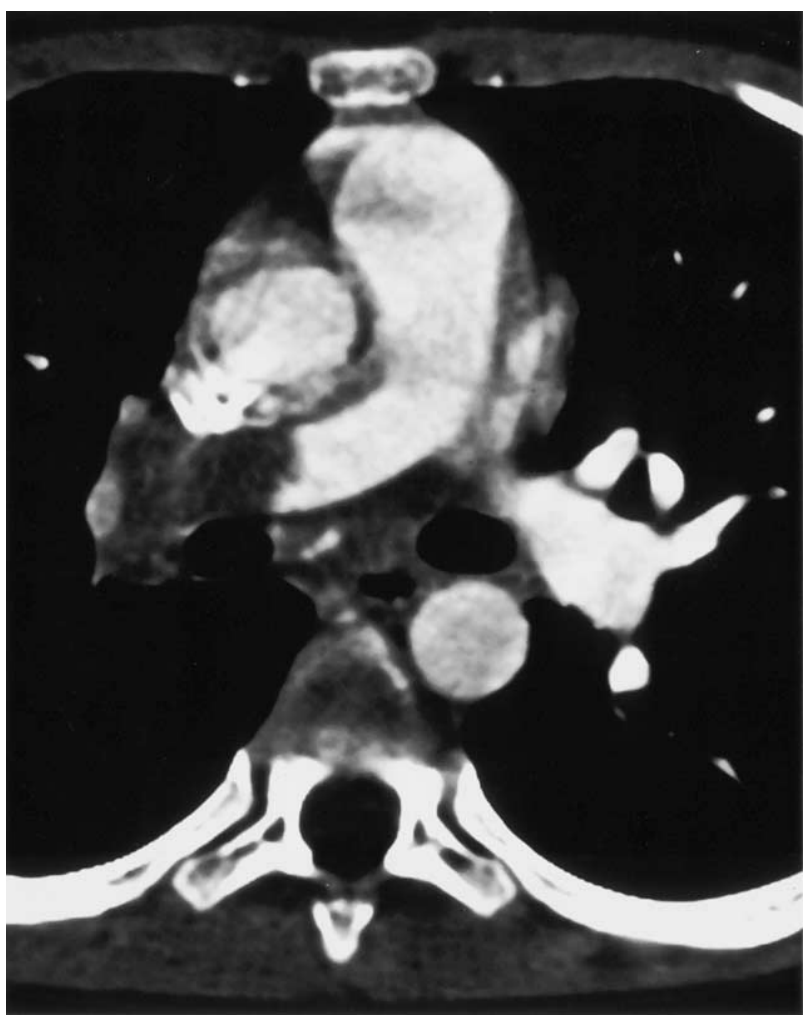

Figure 1. Thoracic computed tomography at admission showing an endoluminal tumor obstructing the retrocaval portion of the right pulmonary artery and extending distally to include the arterial branches of the right upper lobe.

and enlarged supraclavicular and ipsilateral mediastinal lymph nodes. Considering that all previously invasive investigations were inconclusive and that the patient's dyspnea has dramatically worsened over the last 12 hours, we present the patient for surgical exploration and eventually therapy.

Dr Haverich. I would suggest performing the operation through a median sternotomy under cardiopulmonary bypass. This would allow a safe sampling of the regional lymph nodes and biopsy of the tumor mass. The CT extension of the tumor is such that probably a right pneumonectomy with intrapericardial reconstruction of the pulmonary artery trunk would be needed.

Dr Macchiarini. Frankly speaking, I do believe that the operation should be performed through a simple musclesparing right posterolateral thoracotomy without needing cardiopulmonary bypass. The fact that the boy has almost a 1-month history and a tumor mass obstructing the right retrocaval pulmonary artery associated with a rich arterial collateral bronchial network suggests that it could be a slow-growing benign tumor of either the right upper lobe or pulmonary artery. It could be that we might avoid a pneumonectomy by making a bronchial or vascular reconstruc-

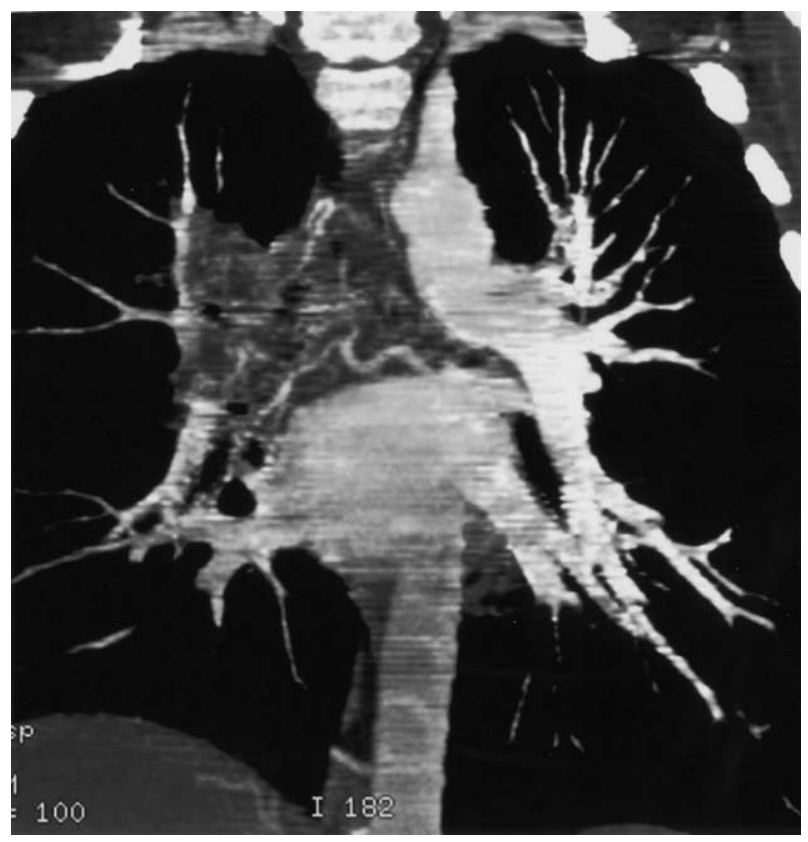

Figure 2. Helical computed tomography scan showing coronar slides of the thoracic cavities. The tumor is located in the upper mediastinum and infiltrates the right pleural cavity. Notice the different density values indicating intense tumor vascularization from the surrounding bronchial arteries.

tion, especially because the pulmonary artery beyond $\mathrm{A} 3$ is free. For these reasons, I would suggest a thoracotomy. The fact that the boy has been deteriorating over the last 12 hours without signs and symptoms of right heart failure also supports my assumption that it could be a slow-growing tumor progressively occluding the right pulmonary artery and resulting in a ventilation/perfusion mismatch.

Dr Haverich. Let's go for the posterolateral thoracotomy.

Dr Macchiarini. The operation was performed through a muscle-sparing posterolateral thoracotomy in the fifth intercostal space. There was a situs solitus without abnormalities in the pleura parietalis and lower and middle lobes. After lysis of some inflammatory adhesions between the mediastinal face of the right upper lobe and the pleura mediastinalis, the right extrapericardial artery was completely dissected. Its outer surface was normal, but palpation showed it filling with a solid mass. After the pericardium was opened and the overlying phrenicus nerve was retracted laterally, the retrocaval and interaorticocaval space were fully mobilized. The CT scans showed an obstruction of the retrocaval portion of the right pulmonary artery (Figure 1). Intraoperatively, the intrapericardial origin of the right pulmonary artery was palpated to investigate whether it was tumor free. It was found that no tumor was present there. Thereafter, the fissure major and all branches of the right pulmonary artery to the middle and lower lobes were dis- 


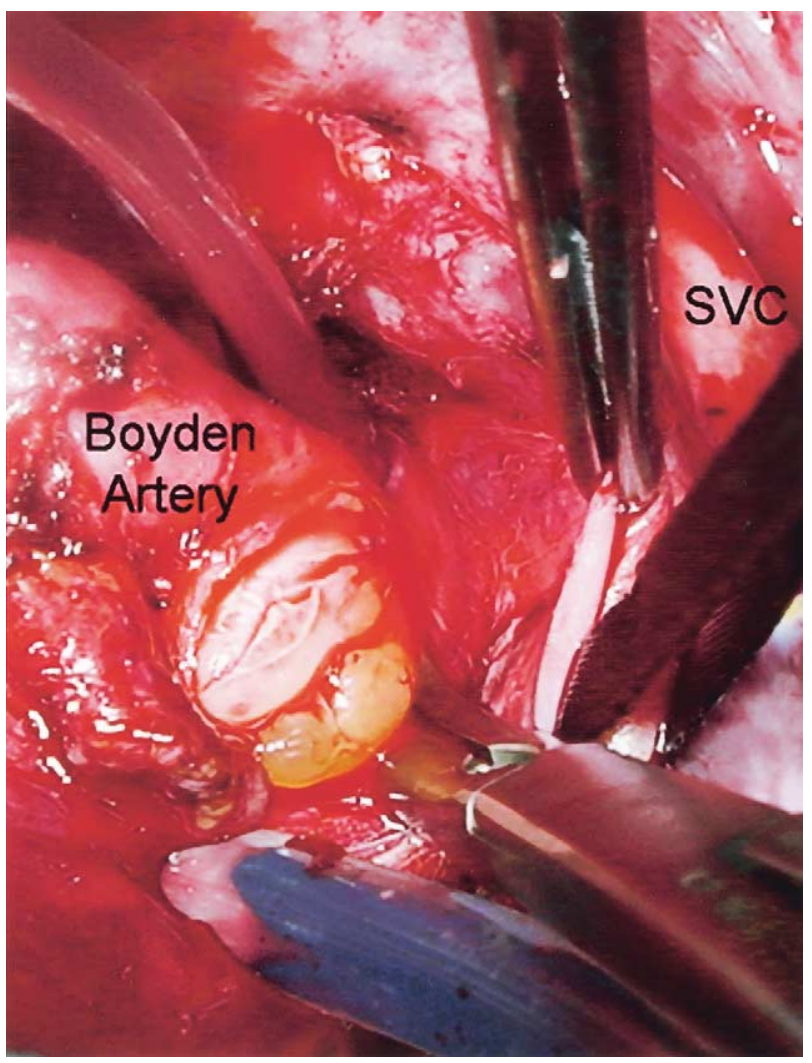

Figure 3. Operative view after arteriotomy on behalf of the Boyden artery and the extraluminal protrusion of a gelatinous tumor. SVC, Superior vena cava.

sected free. This allowed the distal assessment of the disease, showing no tumor beyond the takeoff of the apical segment artery of the right upper lobe. The right upper lobe vein was divided and secured, and after systemic heparinization, the right middle and lower branches of the right pulmonary artery were clamped individually in the Trendelenburg position. After a longitudinal arteriotomy over the exposed ventral surface of the artery, a gelatinous tumor mass protruded outside the lumen (Figure 3). A circumferential dissection plane between the intima of the pulmonary artery and tumor mass was found indicating no infiltration into the vessel wall. Therefore, a Fogarty catheter was placed so that its tip was directed toward the contralateral pulmonary artery. After airing of the balloon, a tumor embolectomy was made, and we were able to completely retract the tumor mass into the arteriotomy extrapericardially. Since the tumor mass was indivisible from the intima of all branches of the right upper lobe pulmonary artery, the decision was made to remove the tumor en bloc with the right upper lobe. Since the adhesion between the artery and right upper lobe bronchus takeoff was undissectable, a right upper lobe sleeve lobectomy was made, followed by endto-end bronchial anastomosis and pericardium-patch closure

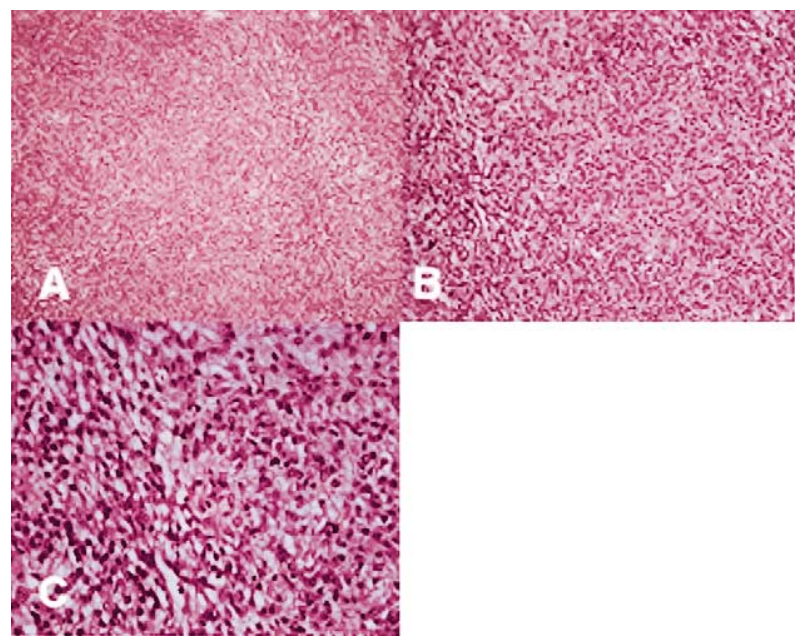

Figure 4. Standard hematoxylin and eosin staining of the tumor samples $(100 \times[A], 200 \times[B]$, and $400 \times[C]$ magnification). Note the dense cords of spindle cells with oval and vesicular nuclei with prominent, sometimes eosinophile, nucleoli. The cytoplasm is rather broad. A rhythmic pattern, sometimes including whorls, can be observed, as well as a multifocal lymphocytic infiltrate. The tumor is very well vascularized. Mitotic count is not elevated, and there is no necrotic tumor tissue.

of the arteriotomy. Frozen sections were all tumor free, and the operation was completed with a radical lymphadenectomy and in a usual fashion. The patient was then transferred to the intensive care unit on mechanical ventilation, where he was extubated on postoperative day 1 .

Dr Freihorst. The patient then came to our normal ward, the pleural drains were removed on day 4 , and he was discharged 14 days after surgery. Since no definitive histologic diagnosis was available, any further decision on postoperative treatment was postponed. To avoid further thromboembolic complications due to the persisting decreased perfusion of the right pulmonary artery, the patient was put on anticoagulant therapy with aspirin and heparin.

Dr Kreipe. The tumor grew within the pulmonary artery and infiltrated the pulmonary parenchyma. Despite the infiltrating tumor growth, there were no frank signs of malignancy, such as necrosis, nuclear atypia, or enhanced mitotic count (Figure 4). Immunohistochemistry of the resected tumor tissue showed a fibrohistiocytic phenotype with a Ki-67 index of 29 (Figure 5). Differential diagnosis included a pulmonary blastoma and an inflammatory pseudotumor, but both alternatives were ruled out on morphologic grounds (Figure 6).

Dr Freihorst. The patient was repeatedly seen in our outpatient clinic after surgery. Two months after surgery, he complained of exertional dyspnea related to a granuloma at the anastomotic site, which was successfully removed endoscopically. Thereafter, the patient remained asymptom- 


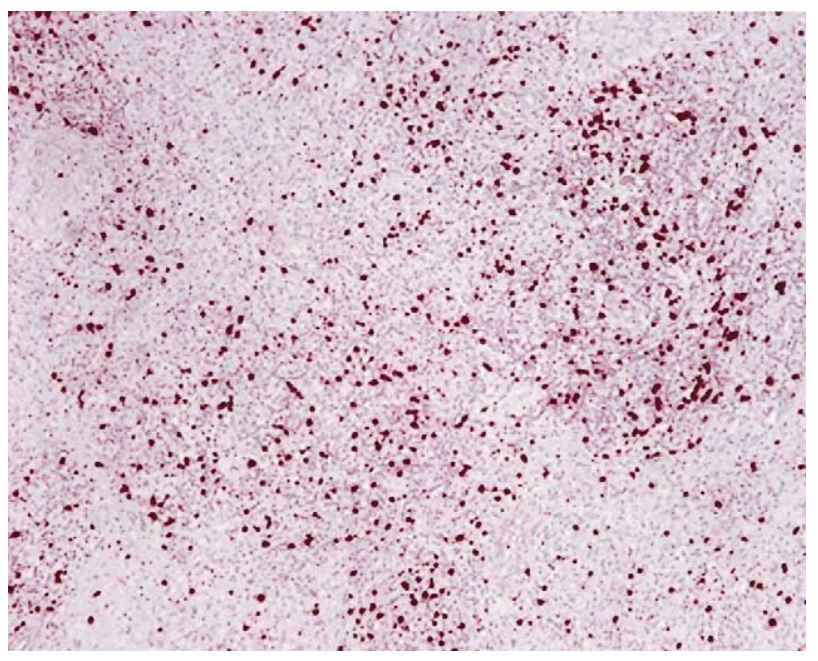

Figure 5. Ki-67-specific staining (100-fold magnification). The growth fraction of tumor cells is between $12 \%$ and $35 \%$.

atic and without evidence of tumor recurrence. Repeated lung functions showed a mild restrictive pattern with a vital capacity between $70 \%$ to $80 \%$ of predicted.

Dr Kreipe. The tumor was reviewed by four additional histopathological experts in the field of soft tissue, pediatric, and pulmonary tumors. None of them came to a definite subclassification of the process and preferred a descriptive diagnosis. The tumor showed no definitive signs of malignancy. The pseudocapsule was intact, and there was no

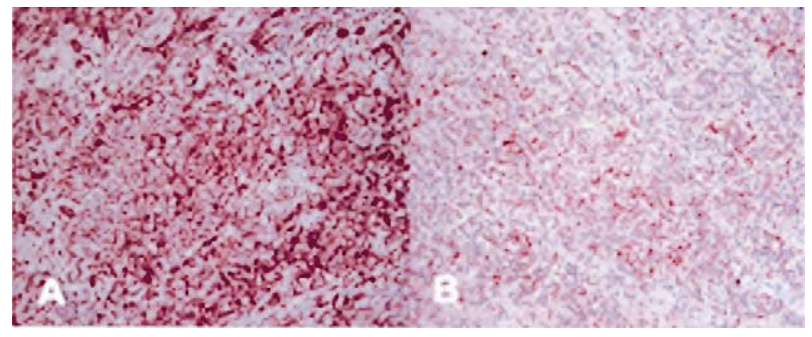

Figure 6. A, The tumor shows a marked reaction to the mesenchymal marker vimentin (vimentin immunostaining; 200-fold magnification). However, there are areas (B) that express keratin (stained with the epithelial marker KL-1; 100-fold magnification).

invasion of vessels. Gross tumor necrosis could not be seen. A somewhat elevated growth fraction can occur in reactive changes or benign tumors as well. Cytology showed only a few atypical tumor cells; there were no giant tumor cells or atypical mitoses. The inflammatory infiltrate suggests an inflammatory pseudotumor, but the dense growth pattern and the monotony of the proliferating spindle cells point to a neoplastic rather than to a reactive genesis of this tumor.

Dr Freihorst, Dr Macchiarini. The pathologic classifications range from benign to any kind of low-grade malignant tumor. Because of this and since no regional nodes were affected by tumor, a decision was made to follow the patient up closely; he is still alive and without evidence of any disease 2.5 years after surgery. 